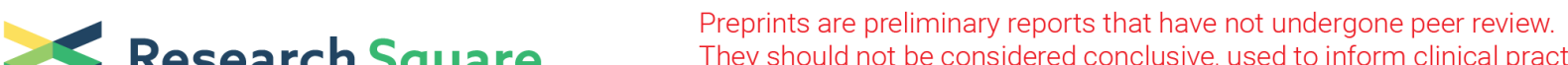 $\begin{array}{ll}\text { Research Square } & \begin{array}{l}\text { They should not be considered conclusive, used to inform clinical practice, } \\ \text { or referenced by the media as validated information. }\end{array}\end{array}$ \\ Tea flavonoids blocking multiple SARS-CoV-2 protein targets judged from molecular docking \\ Lufei Wang \\ MOE Key Laboratory of Contemporary Anthropology, School of Life Sciences, Fudan University \\ Siyao Sang \\ MOE Key Laboratory of Contemporary Anthropology, School of Life Sciences, Fudan University \\ Mingjie Su \\ Human Phenome Institute, Fudan University \\ Simin Wang \\ Innovation Center of Pesticide Research, Department of Applied Chemistry, College of Science, China Agricultural University \\ Hui Li ( $\square$ LHCA@fudan.edu.cn ) \\ MOE Key Laboratory of Contemporary Anthropology, School of Life Sciences, Fudan University
}

\section{Research Article}

Keywords: Severe Acute Respiratory Syndrome Coronavirus 2 (SARS-CoV-2), Severe Acute Respiratory Syndrome Coronavirus 2 (SARS-CoV-2), drug design

Posted Date: December 22nd, 2020

DOl: https://doi.org/10.21203/rs.3.rs-122589/v1

License: (c) (i) This work is licensed under a Creative Commons Attribution 4.0 International License.

Read Full License 


\title{
Tea flavonoids blocking multiple SARS-CoV-2 protein targets judged from molecular docking
}

\author{
Lufei Wang ${ }^{1}$, Siyao Sang ${ }^{1}$, Mingjie Su${ }^{2}$, Simin $\mathrm{Wang}^{3}$, and $\mathrm{Hui}^{\mathrm{Li}}{ }^{1,2,4, *}$ \\ ${ }^{1}$ MOE Key Laboratory of Contemporary Anthropology, School of Life Sciences, Fudan University, Shanghai \\ 200438, China \\ ${ }^{2}$ Human Phenome Institute, Fudan University, Shanghai 200438, China \\ ${ }^{3}$ Innovation Center of Pesticide Research, Department of Applied Chemistry, College of Science, China \\ Agricultural University, Beijing 100193, China \\ ${ }^{4}$ Shanxi Academy of Advanced Research and Innovation, Fudan-Datong Institute of Chinese Origin, Datong \\ 037006, China \\ "Corresponding author: Hui Li. Email: LHCA@fudan.edu.cn
}

\begin{abstract}
Severe Acute Respiratory Syndrome Coronavirus 2 (SARS-CoV-2) has caused Coronavirus Disease 2019 (COVID-19) pandemic. Flavonoids derived Chinese patent medicines has outstanding curative effects for the improvement and treatment of COVID-19. There are numerous studies suggesting that flavonoids-rich tea have antiviral effects. However, bioactive compounds from tea flavonoids with anti-COVID-19 effect, and the potential molecular mechanisms are unclear. In this study, we performed a molecular docking of 468 tea flavonoids and its derivatives with main protease (Mpro), angiotensin-converting enzyme 2 (ACE2), RNA dependent RNA polymerase (RdRp), compared with the positive control drugs of each target. The results suggested that ACE2 and RdRp are the main targets inhibited by tea flavonoids. Q3G Isovitexin, and TF would be considered as the potential candidate compounds of RdRp and ACE2. Our study provides a theoretical basis for further drug design of anti-COVID-19.
\end{abstract}

\section{Introduction}

Coronavirus Disease 2019 (COVID-19) has rapidly spread around the world, causing a pandemic of the infectious pneumonia and enormous damage of social economy. Severe Acute Respiratory Syndrome Coronavirus 2 (SARS-CoV-2) is the causative pathogen of COVID-19, which could be transmitted through droplets expelled during talking, coughing, or sneezing ${ }^{1,2}$. To date (1st December 2020), cumulative numbers of COVID-19 confirmed cases have already exceeded 61 million according to the report of World Health Organization. Systematic autopsy and percutaneous multiple organ biopy discovered that SARSCoV-2 might cause injuries including multiple organs and tissues ${ }^{3}$. Among them, heart failure and acute kidney injury were major complications except pulmonary lesions, even leading to diabetes ${ }^{4}$. Therefore, it is essential to investigate potential components to prevent or treat the multiple organic failure caused by COVID- 19.

Currently, no specific anti-virus drugs or vaccines are available for the treatment of COVID-19. However, greater than $85 \%$ of SARS-CoV-2 infected patients in China had received Traditional Chinese Medicine (TCM) treatment ${ }^{5}$. China had successfully controlled the domestic epidemic through the strict policy and integrative medicine especially traditional Chinese medicine, such as Lianhua Qingwen capsule. Six active compounds of Lianhua Qingwen capsule, belong to flavonoids including quercetin, luteolin, naringenin, kaempferol, wogonin, were identified for treatment of COVID-196. Early in 1985 , naturally occurring flavonoids, in which quercetin reduced intracellular replication of virus, had been reported to possess a variable spectrum of antiviral activity against vertain RNA (RSV, Pf-3, polio) and DNA (HSV-1) viruses acting to inhibit infectivity and/or replication ${ }^{7}$. Shenfu injection was also the recommended Chinese patent medicine for the patients with critical illness of COVID-19. A research suggested that flavonoids derived from Shenfu injection showed favorable binding energy with RNA-dependent RNA polymerase (RdRp) and main protease (Mpro, also known as 3CLpro) ${ }^{8}$. SARS-CoV-2 is an RNA virus with a Mpro which plays an essential role in the production of infectious virions and the replication of SARS$\mathrm{CoV}-2^{9}$. Besides, RdRp is also the central component of coronaviral replication and transcription machinery ${ }^{10}$. Baicalein and quercetin from the flavonoids of Huashi Baidu formula, an auxiliary medicine for the treatment of patients with severe COVID-19, may regulate multiple signaling pathways like TNF signaling pathway through ACE2 ${ }^{11}$. SARS-CoV-2 entries into target cells through the viral structural spike protein binding to the ACE2 receptor ${ }^{12}$. In vitro studies indicated that caflanone, one of the flavonoids, could inhibit SARS-CoV-2 infection ${ }^{13}$. Hence, cumulative evidences suggest that it is a 
promising approach to select the inhibitors of the relevant proteins in SARS-CoV-2 infection from the natural flavonoids based on traditional Chinese medicine (TCM). Meanwhile, Mpro, RdRp, ACE2 have been considered as the potential molecular target for treatment of COVID-19 and anti- SARS-CoV-2 drug discovery.

Tea (Camellia sinensis) as a traditional Chinese medicine contains abundant bioactive compounds such as flavonoids, which has been reported to possess various beneficial effects including cardiovascular-protective, anti-diabetic, immune-regulatory, antiviral effects ${ }^{14,15}$. Tea flavonoids like epigallocatechin gallate (EGCG) have been tested for its antiviral activity against several viruses, which is recognized as a multi-functional bioactive molecule exhibiting anti-inflammatory, antioxidative, antibacterial, antiviral effects ${ }^{16,17}$. One previous study showed inhibition of SARS-CoV 3C-like protease activity by theaflavin-3, 3'-digallate ${ }^{18}$. Recently, epigallocatechin-3-gallate and theaflavin-3,3'-digallate derived from tea flavonoids had a significant interaction with the receptors of SARS-CoV-2, which suggested the use of tea flavonoids as potential candidates in prophylaxis and treatment of COVID-19 ${ }^{17,19}$. In vitro study demonstrated epigallocatechin-3-gallate $\left(\mathrm{IC}_{50}: 7.58 \mu \mathrm{g} / \mathrm{ml}\right)$ and theaflavin $\left(\mathrm{IC}_{50}: 8.44 \mu \mathrm{g} / \mathrm{ml}\right)$ showed inhibitory activity against the SARS-CoV-2 Mpro in a dose-dependent manner. Moreover, different flavonoids have been investigated for their potential antiviral activities and several of them shown significant antiviral properties in vitro and in vivo ${ }^{20}$.

Network pharmacology is a promising approach to identify potential novel drugs or targets based on interaction between multi-compounds and multi-targets ${ }^{21,22}$. Molecular docking, a reliable method for drug discovery, is widely used to investigation of novel compounds against disease to predict ligand-protein interactions pose and molecular mechanism ${ }^{23,24}$. To select new inhibitors of the relevant proteins in SARS-CoV-2 infection and unveil the mechanism of compounds-targets interaction, we performed the molecular docking to investigate the tea flavonoids against Mpro, RdRp, ACE2 and elucidate the molecular mechanism. The flow scheme of our study was shown in Figure 1.

\section{Results}

\section{Docking score of compounds-targets interaction}

In the 468 tea flavonoids, 121 compounds with molecular weight below $500 \mathrm{~g} / \mathrm{mol}$ were collected for molecular docking. The ligand-protein complex will be more stable when higher the docking score (DS) absolute value (|DS|) because the DS was negative. $|\mathrm{DS}|$ of the compounds-targets interaction were shown in Figure 2. The higher $|\mathrm{DS}|$ was observed in RdRp (mean \pm sd, $7.31 \pm 1.18$ ), while lower $|\mathrm{DS}|$ was shown in Mpro (mean \pm sd, $6.63 \pm 0.72$ ). The RdRp and ACE2 (mean \pm sd, $7.51 \pm 0.98$ ) were significantly higher $|\mathrm{DS}|$ compared with Mpro. Therefore, preliminary analysis indicated RdRp and ACE2 could be the main targets for tea flavonoids against COVID-19.

\section{Network analysis of compounds-targets interaction}

To further investigate the interaction between tea flavonoids and targets, we established a network model of compoundstargets interaction. The compounds-targets interaction network was shown in Figure 3. 90 nodes (87 compounds and 3 targets) and 192 edges were contained in network model. There are 22 compounds with single target (Supplementary Table 1) and 65 compounds with multiple targets, which reveals that tea flavonoids exhibit a synergistic effect for anti-COVID-19.

According to the perspective of targets, ACE2 has a highest degree value of 82, degree value of RdRp is 68 and Mpro has the degree value of 45. In addition, the numbers of single-target compounds are different for different targets. Among them, 16 single-target compounds were interacted with ACE2, 5 single-target compounds were interacted with RdRp and a singletarget compound is interacted with Mpro (Supplementary Table 1). These single target compounds were shown outside the circle.

According to degree value analysis of tea flavonoids, 40 tea flavonoids interacting with 3 targets (Supplementary Table 2) were collected and the top 10 tea flavonoids of DS for each target were identified (Table 2). Although 9 tea flavonoids of the top 10 compounds in Mpro may interact with 3 targets, DS of tea flavonoids interacting with Mpro was higher than the positive control lopinavir (Supplementary Table 2, Table 1, Table 2). There were 6 compounds of the top 10 tea flavonoids could interact with 3 targets in RdRp, in which DS of 5 tea flavonoids is lower than the positive control remdesivir (Supplementary Table 2, Table 1, Table 2). For ACE2, 6 tea flavonoids with degree value of 3 in the top 10 compounds were lower than the positive control hydroxychloroquine (Supplementary Table 2, Table 1, Table 2).

\section{Identification of the key tea flavonoids}

To further identify the tea flavonoids of each target, ADME was predicted based on molecular structure, which represents absorption, distribution, metabolism, and excretion ${ }^{25}$. Drug like (DL) and bioavailable score (BS) are a qualitative character to 
describe the physical and chemical properties of drugs and ADME properties ${ }^{26} .6$ overlapping compounds from the top 10 tea flavonoids in each target also were illustrated. Among them, Quercetin 3-glycosides (Q3G), Epigallocatechin 3-O-caffeate, Cyanidin 3-glycosides and Delphinidin 3-glycosides may interact with 3 targets, while 4',5,7-trihydroxyflavanone 7-Ofructoside (S)-form (TF) and isovitexin could interact with 2 targets (RdRp and ACE2). Then, DL and BS of these tea flavonoids were predicted. However, cyanidin 3-glycosides is removed due to its DL is less than 0.18 . Among the overlapping compounds, DS of Q3C, isovitexin and TF are lower than that of the corresponding positive control drugs remdesivir and hydroxychloroquine, and their DL and BS satisfied the threshold. Above results suggests that ACE2 and RdRp are the important targets interacted with tea flavonoids, which was consistent with the previous analysis of the compounds-targets interaction. Meanwhile, Q3C, isovitexin and TF were identified for further analysis.

\section{Analysis of interaction between the selected tea flavonoids with targets}

To further analysis binding modes and sites between tea flavonoids and targets, 3D active pockets and 2D docking interaction of positive control drugs and tea flavonoids with targets were compared. RdRp is essential for the replication and transcription of SARS-CoV-2, which is a target of the antiviral drug remdesivir ${ }^{27}$. Remdesivir is covalently incorporated into the primer strand at the first replicated base pair, and terminates chain elongation ${ }^{28}$, which is considered as the positive control drug. TMDB-00229, isovitexin, Q3G, TF and the positive control remdesivir with RdRp were shown in Figure 4. TMDB-00229 with a lowest DS of -11.83 was the optimum compound in all tea flavonoids (Table 2). Interaction of DS of TMDB-00229, isovitexin, Q3G and TF were lower than that of remdesivir (Table 1, Table 3). Although TMDB-00229 had the highest DS, hydrogen bond was not observed (Figure 4B). Tyr619 had a strong hydrogen interaction with remdesivir and isovitexin (Figure 4A, 4C). Asp618 formed a strong hydrogen interaction with isovitexin, Q3C, TF (Figure 4C, 4D, 4E). The amino acid residues Glu811 was involved in formation of hydrogen bond with Q3C (Figure 4D). TF also formed a hydrogen bond with Asp760 (Figure 4E). Above analysis indicated isovitexin, Q3C and TF could be considered as potential inhibitors of RdRp.

ACE2 is widely expressed in multiple human organs like lungs, cardiovascular system, kidneys, etc ${ }^{29}$. ACE2 was reported as an entry receptor for SARS-CoV-2, which could bind with the viral spike protein ${ }^{30}$. Hydroxychloroquine is thought to weaken the terminal glycosylation of the ACE2 ${ }^{31}$. It is regard as a positive control drug. TMDB-00174 with a lowest DS of -10.03 was the optimum compound in all tea flavonoids (Table2). DS of other selected tea flavonoids including isovitexin, Q3G, TF were lower than that of positive control hydroxychloroquine, which suggested 4 tea flavonoids had strong binding capacity with ACE2(Table 1, Table 3). Arg518 could form hydrogen bond with TMDB-00174 and hydroxychloroquine (Figure 5A, 5B). TMDB-00174 and Q3G had a $\pi$-H interaction with ACE2 (Figure 5B, 5D). The amino acid residuesThr445 and His345 formed two strong hydrogen bond with TMDB-00174 (Figure 5B). Pro346 could form two strong hydrogen bond with isovitexin (Figure 5C). TF formed a hydrogen bond with Glu375 (Figure 5E). Our data demonstrated TMDB-00174, isovitexin, Q3G and TF would be candidate inhibitors of ACE2. Taken together, Q3G, isovitexin and TF could be a lead compounds for prevention of COVID-19 based on interaction and DS.

\section{Discussion}

As the weather turned colder, the second wave of COVID-19 should be attracted attention by all over the world. Remarkably, China, as a major country with a population of more than 1.3 billion, has effectively managed the epidemic outbreak in the short term. Except the strict outbreak-contained measures, TCM played critical role in the prevention and treatment of COVID-19. Research on the clinical Chinese patent medicine demonstrated that flavonoids derived Chinese herb are considered as effective inhibitors for the target of SARS-CoV-2. Therefore, it is a reliable approach to screen inhibitors from herb-derived flavonoids. Flavonoids derived Chinese herb have antiviral effect as well as multiple organ protection ${ }^{14}$, which could be regard as candidate compounds for multiple organ failure caused by COVID-19. Tea contained abundant flavonoids has been proven to be effective for anti-virus and multiple organ protection. In this study, we conducted a molecular docking to screen potential anti-COVID-19 compounds from 468 tea flavonoids. DS-based compounds-targets network was constructed. We obtained 22 single-target compounds and 40 compounds with 3 targets. Significant interaction of two targets (ACE2 and RdRp) and candidate tea flavonoids (especially Q3G, isovitexin, TF, and more) were analyzed. The results of the research demonstrated the effectiveness of tea flavonoids in the treatment of COVID-19 from a bioinformatics perspective, and may also promote target drug design and basic research on SARS-CoV-2 infection.

Our study obtained several compounds of tea flavonoids according DS of molecular docking. Although DS of top 10 in Mpro was lower than the positive control lopinavir, recent study indicated that green tea polyphenols (especially epigallocatechin gallate, epicatechingallate and gallocatechin-3-gallate) were known to be used as potential inhibitors against Mpro. The top 10 tea flavonoids of Mpro in our study also included epigallocatechin gallate, which suggested epigallocatechin gallate would be a promising candidate drug. Recent study indicated that EGCG could counteract hyper-inflammation growing in COVID-19 because its antiviral, anti-sepsis, anti-fibrotic effect and reduction in expression and signaling of many inflammatory mediators (like NF- $\mathrm{kB})^{17}$. Remdesivir has been a strong drug candidate against COVID-19 through inhibiting RdRp ${ }^{10,32}$. DS 
of the top 7 tea flavonoids in RdRp was higher than remdesivir. Interaction between tea flavonoids and RdRp was very similar to remdesivir. Simultaneously, RdRp also is a major target of tea flavonoids.

ACE2, a target of hydroxychloroquine, is a homologue of ACE that catalyzes the conversion of Angiotensin II into Angiotesin 1-7, which induces vasodilation, anti-fibrotic, anti-proliferative and anti-inflammatory effects ${ }^{33}$. Chloroquine and Hydroxychloroquine have been confirmed in vitro these drugs might inhibit SARS-CoV-2 by elevating the endosomal $\mathrm{pH}$, and alter ACE-2 terminal glycosylation there by leading to the interruption of virus receptor binding ${ }^{34}$. However, the Food and Drug Administration of USA declared that accompanies the drug to state that co-administration of remdesivir and chloroquine phosphate or hydroxychloroquine sulfate may result in reduced antiviral activity of remdesivir. Thus, it is essential to discover bioactive compounds against ACE2 for replacing hydroxychloroquine. In this study, we identified a major target ACE2 for anti-COVID-19 tea flavonoids. Meanwhile, our data suggested that DS of top 10 tea flavonoids is higher than hydroxychloroquine. Interaction of tea flavonoids with ACE2 have more hydrogen bonds to form stable ligandtarget complex, compared with that of hydroxychloroquine. The single-cell RNA sequencing (scRNA-seq) data identified the organs at risk like heart and kidney based on ACE2 expression levels in some cell types of different organs ${ }^{35}$. A genomewide association study identified a kidney failure-related 3p21.31 gene cluster as a genetic susceptibility locus in patients with COVID-19 with respiratory ${ }^{36}$. Moreover, a latest study demonstrated the presence of SARS-CoV-2 RNA and protein in anatomically distinct regions of the nasopharynx and brain $^{37}$. Cumulative evidence indicate that a heart-brain-kidney network $^{38}$ may be associated with SARS-CoV-2 infection. Interestingly, heart and kidney belong to little lunar (ShaoYin) meridian based on meridian theory of TCM. A recent study done in our lab revealed that flavonoids-rich yellow tea could activate little lunar meridian, that is a directional flow of body fluid, which provide a strategy of drug discovery ${ }^{39}$. Therefore, yellow tea with abundant flavonoids could be considered as a compound Chinese medicine for prevention of COVID-19 and tea flavonoids would be a promising drug candidate.

In this pharmacology network-based study, we investigated the potential therapeutic mechanisms of the tea flavonoids against COVID-19. The results highlight Q3G, isovitexin and TF would be considered as the potential drug candidate. Additionally, the RdRp and ACE2 were main potential target for COVID-19 treatment in tea flavonoids. In view of the limitations of virtual screening results, further experiments in vivo and in vitro are needed to verify the results of this study so as to provide experimental basis for the research and development of antiviral natural drug.

\section{Conclusion}

Our study uncovered the potential bioactive compounds (especially Q3C, isovitexin, TF, and more) of tea flavonoids against COVID-19 by employing pharmacology network and molecular docking-based virtual screening analyses. We believe that these findings may aid the global fight against the novel coronavirus pneumonia epidemic.

\section{Methods}

\section{Collection of tea flavonoids compounds and anti-COVID-19 targets}

468 compounds of tea flavonoids and its derivatives were retrieved from Tea Metabolome Database (TMDB, http://pcsb.ahau.edu.cn:8080/TCDB/f). 121 compounds were identified based on molecular weight of small molecules $(<$ $500 \mathrm{~g} / \mathrm{mol}$ ). The structures of these compounds were sketched in ChemDraw saved in mol format, then minimized in MOE by applying a AMBER 10 force field for further research.

The information of clinical drugs and protein targets for the prevention and treatment of COVID-19 (Table 1) was gained from DrugBank (https://go.drugbank.com/). The crystal structure of each protein targets with its embedded ligands (Table 1) was obtained from the Protein Data Bank (http://www.rcsb.org/pdb).

\section{Molecular docking}

Molecular docking analysis was performed using Molecular Operating Environment. The binding site was identified based on the embedded ligands site of crystal structure of each target. Each target was protonated 3D at physiological $\mathrm{pH}$ prior to docking and removed water, then docked with 121 compounds. The best conformation with a low DS will be gained for next analysis. The London dG scoring function estimates the free energy of binding of the ligand from a given pose. Value of docking score was used to evaluate binding ability. Ligand-protein complex with the lower DS was more stable.

\section{Construction of compounds-targets interaction network based on DS}

Network interaction model of compounds-targets was constructed and visualized by Cytoscape 3.8.1 software. Compounds and targets were shown by different colored square nodes in the network model. The compounds and targets will be connected if the DS absolute value is more than 7 . The red edge denote a strong binding capacity $(|\mathrm{DS}| \geq 8)$, while the black edge denotes a good binding capacity $(8 \geq|\mathrm{DS}| \geq 7)$. Degree, topological parameters were calculated by Cytoscape. The 
significant difference of DS absolute value of tea flavonoids for each target was tested with pairwise. t. test (p. adjust. Method = "fdr"). The boxplot was generated by ggplot2 package in R-3.6.2.

\section{Prediction of ADMET properties.}

ADME refers to the absorption, distribution, metabolism and excretion of the compounds, which is a important parameters to evaluate pharmacological effects. According to the favorable level, drug-like score (DL) $\geq 0.18$ and bioavailability score (BS) $\geq 0.17$ are often assigned as the criteria to evaluate active compounds. DL and BS were calculated by the molsoft website (https://www.molsoft.com/mprop/) and SwissADME online tool (http://www.swissdock.ch/), respectively.

\section{References}

1. Zheng, J. SARS-CoV-2: an emerging coronavirus that causes a global threat. International journal of biological sciences 16, 1678-1685, (2020).

2. Wiersinga, W. J., Rhodes, A., Cheng, A. C., Peacock, S. J. \& Prescott, H. C. Pathophysiology, Transmission, Diagnosis, and Treatment of Coronavirus Disease 2019 (COVID-19): A Review. Jama 324, 782-793, (2020).

3. Bian, X.-W. Autopsy of COVID-19 victims in China. National Science Review 7, 1414-1418, (2020).

4. Mallapaty, S. Mounting clues suggest the coronavirus might trigger diabetes. Nature 583, 16-17, (2020).

5. Tian, T. et al. Tea consumption and risk of stroke in Chinese adults: a prospective cohort study of 0.5 million men and women. Am J Clin Nutr 111, 197-206, (2020).

6. Xia, Q.-D. et al. Network pharmacology and molecular docking analyses on Lianhua Qingwen capsule indicate Akt1 is a potential target to treat and prevent COVID-19. Cell Proliferation n/a, e12949-e12961, (2020).

7. Kaul, T. N., Middleton, E., Jr. \& Ogra, P. L. Antiviral effect of flavonoids on human viruses. J Med Virol 15, 71-79, (1985).

8. Li, X. et al. Chemical composition and pharmacological mechanism of Shenfu decoction in the treatment of novel coronavirus Pneumonia (COVID-19). Drug development and industrial pharmacy, 1-32, (2020).

9. Zumla, A., Chan, J. F., Azhar, E. I., Hui, D. S. \& Yuen, K. Y. Coronaviruses - drug discovery and therapeutic options. Nature reviews. Drug discovery 15, 327-347, (2016).

10. Gao, Y. et al. Structure of the RNA-dependent RNA polymerase from COVID-19 virus. Science 368, 779-782, (2020).

11. Tao, Q. et al. Network pharmacology and molecular docking analysis on molecular targets and mechanisms of Huashi Baidu formula in the treatment of COVID-19. Drug development and industrial pharmacy 46, 1345-1353, (2020).

12. Wiersinga, W. J., Rhodes, A., Cheng, A. C., Peacock, S. J. \& Prescott, H. C. Pathophysiology, transmission, diagnosis, and treatment of Coronavirus Disease 2019 (COVID-19): a review. Jama 324, 782-193, (2020).

13. Ngwa, W. et al. Potential of flavonoid-inspired phytomedicines against COVID-19. Molecules (Basel, Switzerland) 25, 2707-2716, (2020).

14. Bondonno, N. P. et al. Flavonoid intake is associated with lower mortality in the Danish Diet Cancer and Health Cohort. Nature Communications 10, 3651-3660, (2019).

15. Tang, G. Y. et al. Health Functions and Related Molecular Mechanisms of Tea Components: An Update Review. International journal of molecular sciences 20, 6196-6233, (2019).

16. Chacko, S. M., Thambi, P. T., Kuttan, R. \& Nishigaki, I. Beneficial effects of green tea: A literature review. Chinese Medicine 5, 13-21, (2010).

17. Mhatre, S., Srivastava, T., Naik, S. \& Patravale, V. Antiviral activity of green tea and black tea polyphenols in prophylaxis and treatment of COVID-19: A review. Phytomedicine : international journal of phytotherapy and phytopharmacology, 153286-153293, (2020).

18. Chen, C. N. et al. Inhibition of SARS-CoV 3C-like protease activity by theaflavin-3,3'-digallate (TF3). Evid Based Complement Alternat Med 2, 209-215, (2005).

19. Menegazzi, M. et al. Protective Effect of Epigallocatechin-3-Gallate (EGCG) in Diseases with Uncontrolled Immune Activation: Could Such a Scenario Be Helpful to Counteract COVID-19? International journal of molecular sciences 21, 5171-5189, (2020).

20. Zakaryan, H., Arabyan, E., Oo, A. \& Zandi, K. Flavonoids: promising natural compounds against viral infections. Arch Virol 162, 2539-2551, (2017).

21. Yuan, H. et al. How Can Synergism of Traditional Medicines Benefit from Network Pharmacology? Molecules (Basel, Switzerland) 22, 1135-1153, (2017).

22. Zhang, Q. et al. Natural formulas and the nature of formulas: Exploring potential therapeutic targets based on traditional Chinese herbal formulas. PloS one 12, e0171628-e0171641, (2017).

23. Pinzi, L. \& Rastelli, G. Molecular docking: shifting paradigms in drug discovery. International journal of molecular sciences 20, 4331-4353, (2019).

24. Saikia, S. \& Bordoloi, M. Molecular Docking: Challenges, Advances and its Use in Drug Discovery Perspective. Current drug targets 20, 501-521, (2019).

25. Bhhatarai, B., Walters, W. P., Hop, C. E. C. A., Lanza, G. \& Ekins, S. Opportunities and challenges using artificial 
intelligence in ADME/Tox. Nature Materials 18, 418-422, (2019).

26. Zhang, X. et al. Network pharmacology based virtual screening of active constituents of Prunella vulgaris L. and the molecular mechanism against breast cancer. Scientific Reports 10, 15730, (2020).

27. Hillen, H. S. et al. Structure of replicating SARS-CoV-2 polymerase. Nature 584, 154-156, (2020).

28. Yin, W. et al. Structural basis for inhibition of the RNA-dependent RNA polymerase from SARS-CoV-2 by remdesivir. Science 368, 1499, (2020).

29. Gheblawi, M. et al. Angiotensin-Converting Enzyme 2: SARS-CoV-2 Receptor and Regulator of the ReninAngiotensin System. Circulation Research 126, 1456-1474, (2020).

30. Lan, J. et al. Structure of the SARS-CoV-2 spike receptor-binding domain bound to the ACE2 receptor. Nature 581, 215-220, (2020).

31. Boulware, D. R. et al. A Randomized Trial of Hydroxychloroquine as Postexposure Prophylaxis for Covid-19. New England Journal of Medicine 383, 517-525, (2020).

32. Hendaus, M. A. Remdesivir in the treatment of coronavirus disease 2019 (COVID-19): a simplified summary. Journal of biomolecular structure \& dynamics, 1-6, (2020).

33. Anguiano, L., Riera, M., Pascual, J. \& Soler, M. J. Circulating ACE2 in Cardiovascular and Kidney Diseases. Current medicinal chemistry 24, 3231-3241, (2017).

34. Nimgampalle, M., Devanathan, V. \& Saxena, A. Screening of Chloroquine, Hydroxychloroquine and its derivatives for their binding affinity to multiple SARS-CoV-2 protein drug targets. Journal of biomolecular structure \& dynamics, 1$13,(2020)$.

35. Zou, X. et al. Single-cell RNA-seq data analysis on the receptor ACE2 expression reveals the potential risk of different human organs vulnerable to 2019-nCoV infection. Frontiers of Medicine 14, 185-192, (2020).

36. Ellinghaus, D. et al. Genomewide Association Study of Severe Covid-19 with Respiratory Failure. New England Journal of Medicine 383, 1522-1534, (2020).

37. Meinhardt, J. et al. Olfactory transmucosal SARS-CoV-2 invasion as a port of central nervous system entry in individuals with COVID-19. Nature Neuroscience, (2020).

38. Fujiu, K. et al. A heart-brain-kidney network controls adaptation to cardiac stress through tissue macrophage activation. Nature Medicine 23, 611-622, (2017).

39. Jin, W. et al. Infrared imageries of human body activated by teas indicate the existence of meridian system. Quantitative Biology, (2020). 


\section{Acknowledgements}

This work was supported by National Key R\&D Program of China (2020YFE0201600), B\&R Joint Laboratory of Eurasian Anthropology (18490750300), Shanghai Ziranerran Chinese Medicine Development Foundation, Natural Science Foundation of China (31671297, 91731303).

\section{Author contributions statement}

L.F.W. wrote the paper, analyzed the data, drew the figures. L.F.W. and S.Y.S conducted experiment. S.Y.S, M.J.S and H.L revised the paper. S.M.W provided software and technical assistance. H.L. and L.F.W. conceived the experiments.

\section{Competing interests}

The authors have declared no conflicts.

\section{Additional information}

Correspondence and requests for materials should be addressed to H.L.

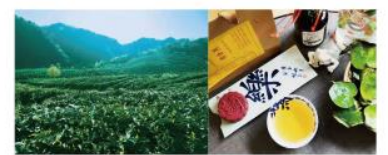

Camellia sinensis

Collection and screening<smiles>c1ccc(C2CCc3ccccc3O2)cc1</smiles>

121 compounds from tea flavonoids
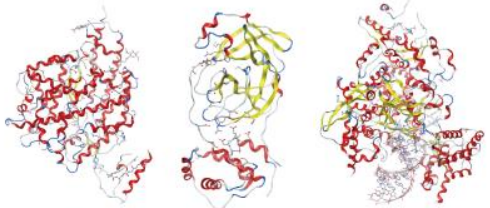

COVID-19-related protein target

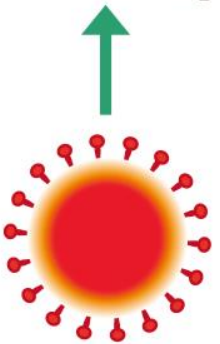

SARS-CoV-2

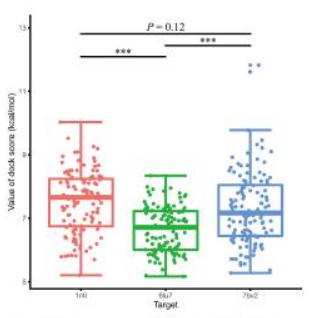

Docking Score (binding energy)

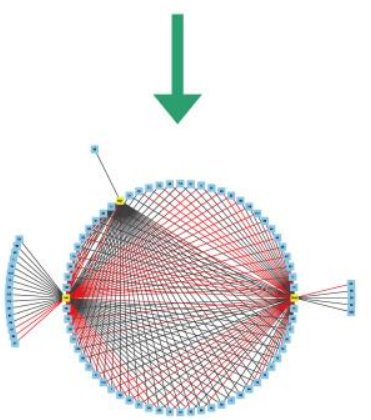

Compounds-targets network

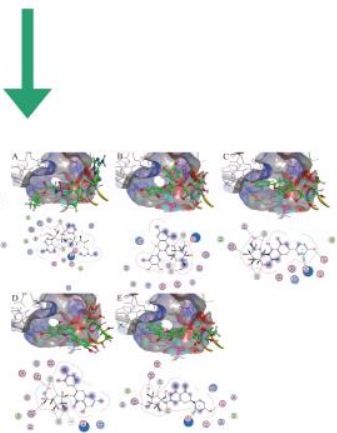

The best conformation and main target

Figure 1. Flow scheme of exploring the inhibitors against multiple SARS-CoV-2 targets 


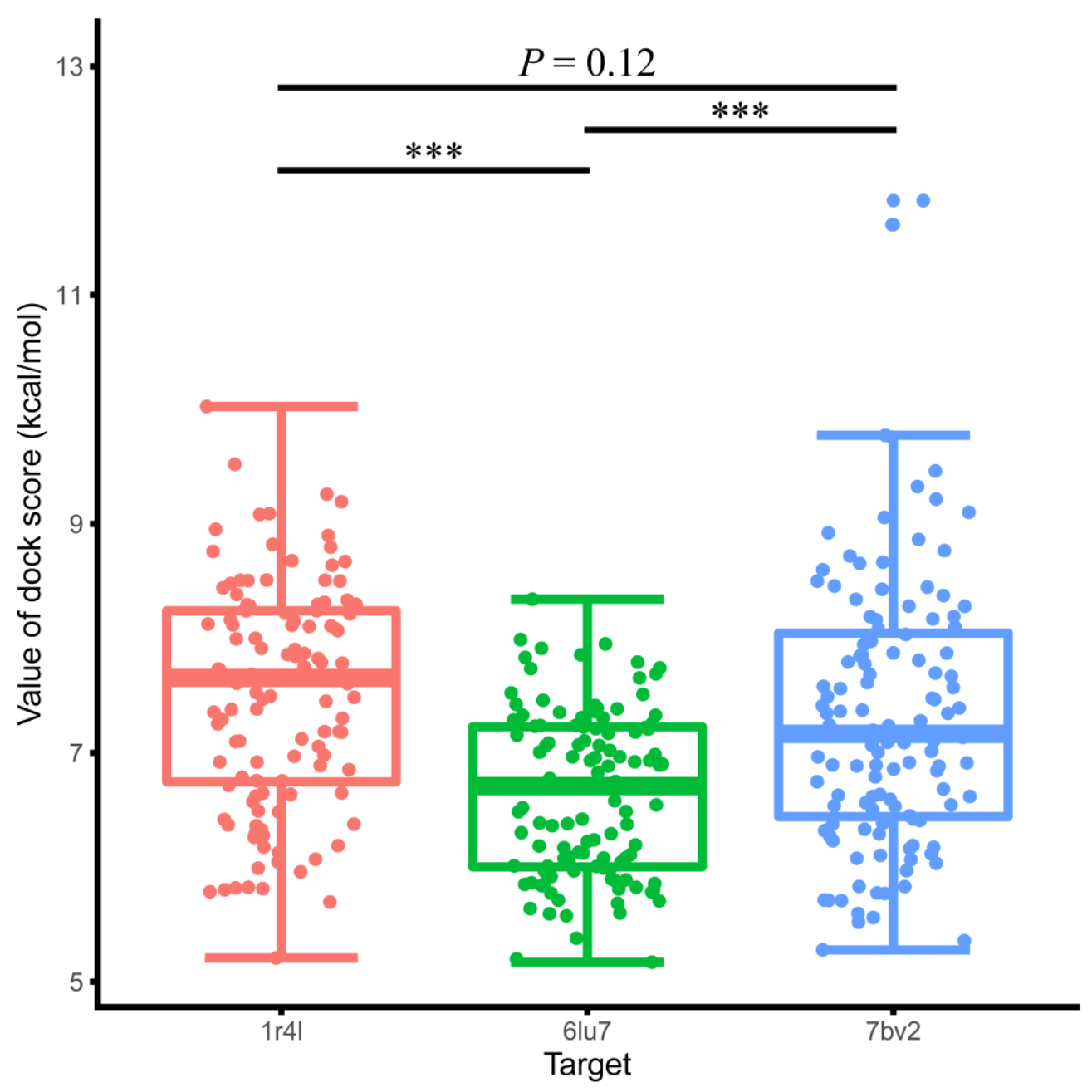

Figure 2. DS absolute value of the compounds-targets interaction. The minimum and maximum values are directly observed from the boxplot; the boxplot center is the median; the boxplot edges represent the 25 th and 75 th percentiles. Pairwise. t. test (p. adjust. method $=$ "fdr"). *: $\mathrm{p}<0.05 ; * *: \mathrm{p}<0.01 ; * * * \mathrm{p}<0.001$. 


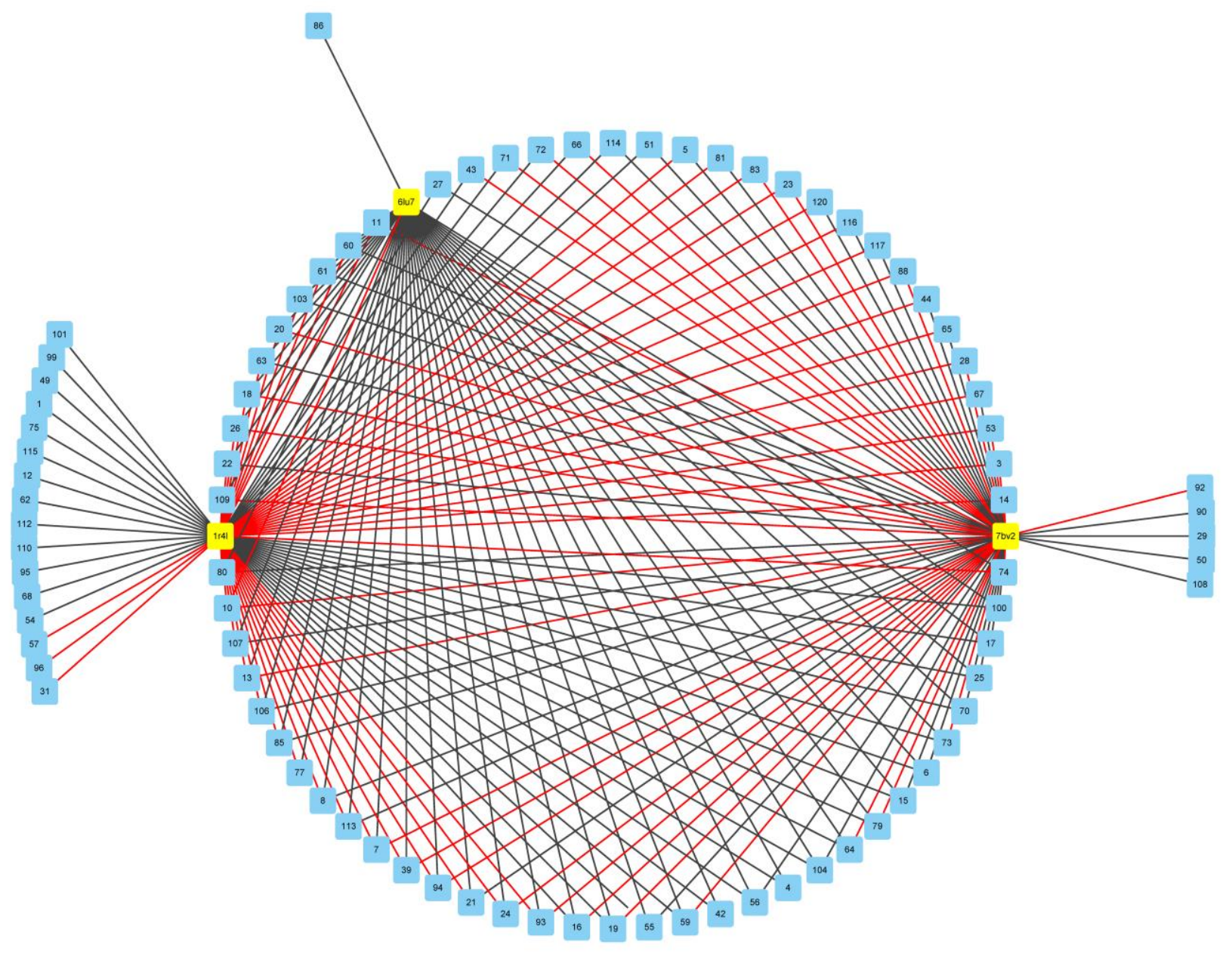

Figure 3. The network model of the compounds-targets interaction. The blue square nodes represent the tea flavonoids, the yellow square nodes represent the protein targets. The red edge denote a strong binding capacity $(|\mathrm{DS}| \geq 8)$, while the black edge denotes a good binding capacity $(8 \geq|\mathrm{DS}| \geq 7)$. 

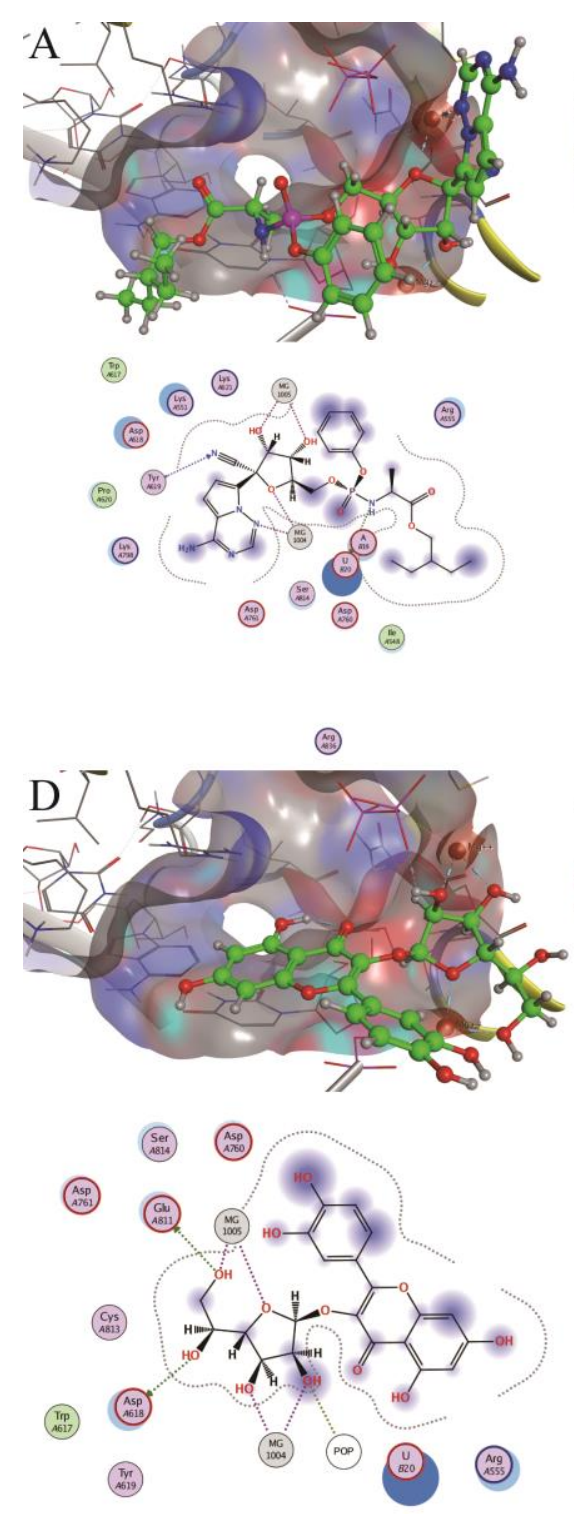
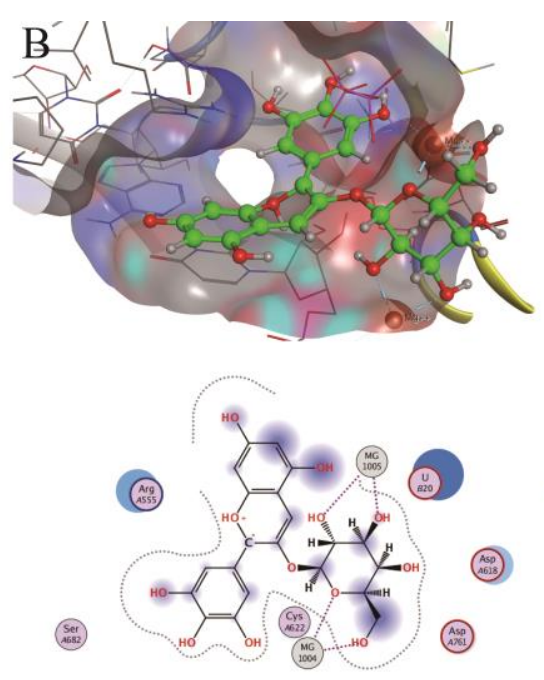

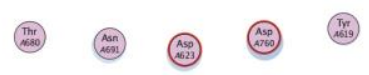

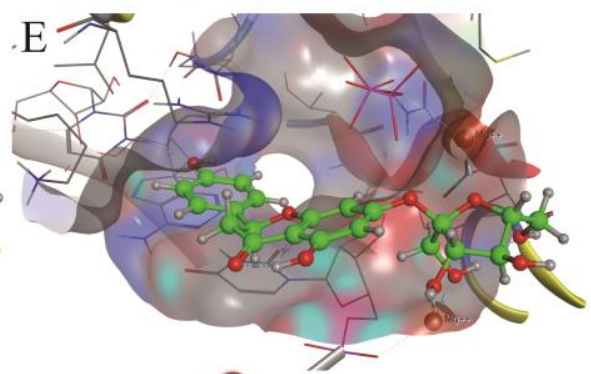

(슈유)

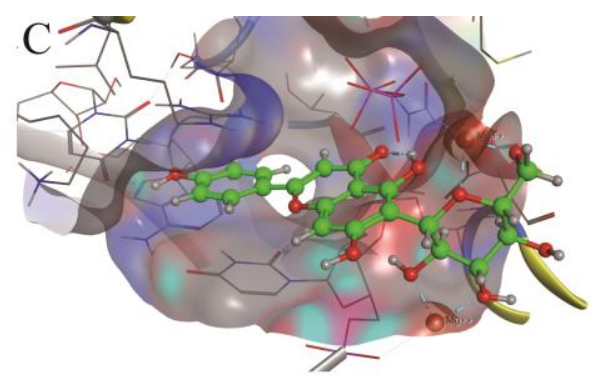

(ii:

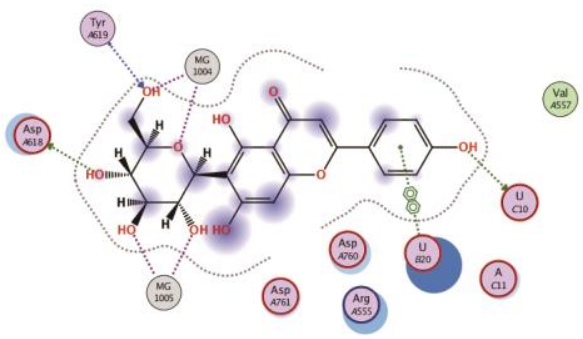

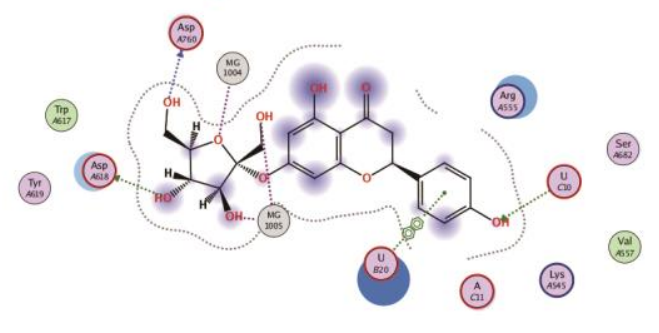

Figure 4. 3D active pocket and 2D interaction of identified optimum conformation against RdRp (7bv2). (A) Remdesivir (B) TMDB-00229 (C) Isovitexin (D) Q3G (E) TF. 


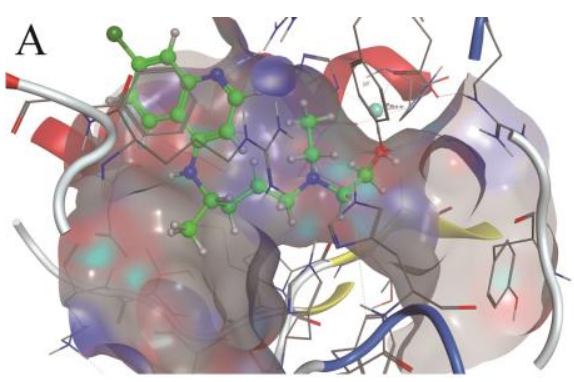

(iiii)

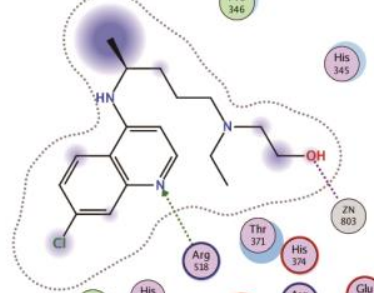

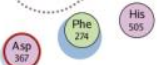

영 쥬유 : : :

(iii) (iii) (iii)

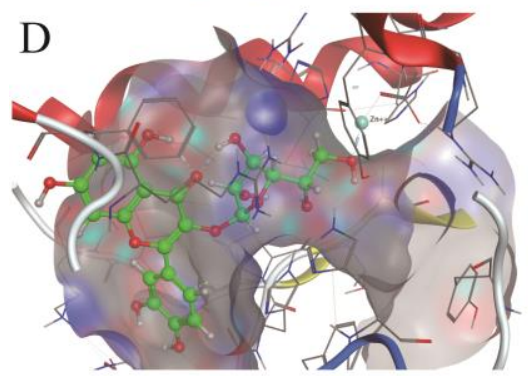

(:)

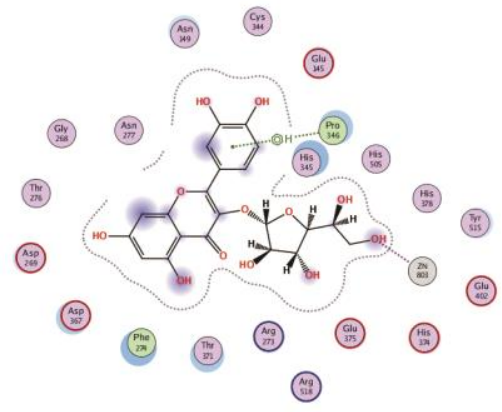

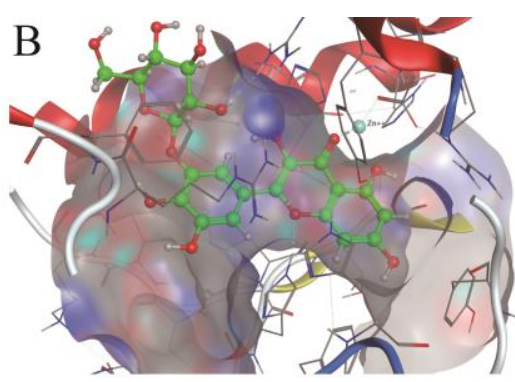

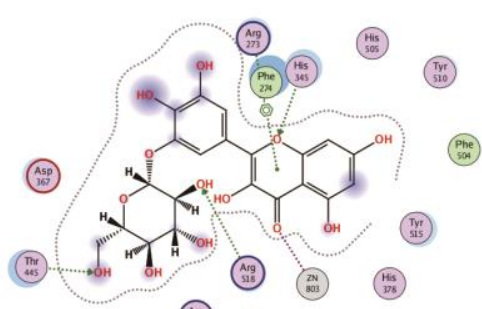

(iiin)

(12:)

(ii:) (::0)

(15)

(i:3) (iii) 궁

(주)

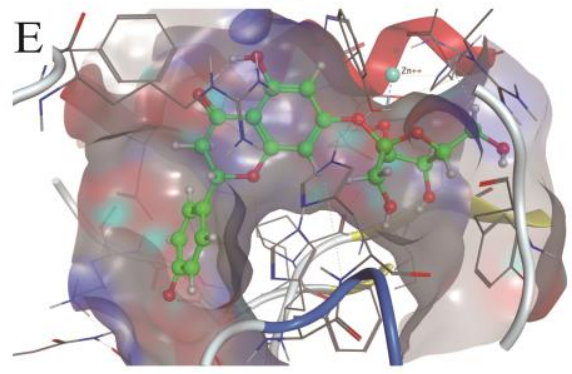

;:)

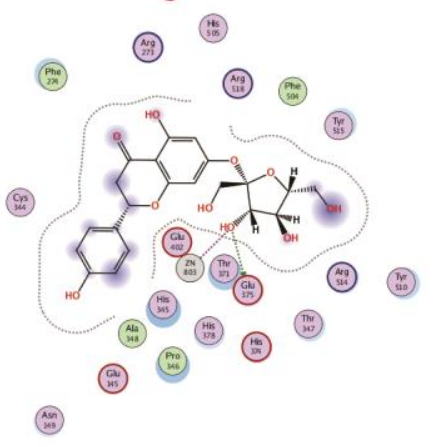

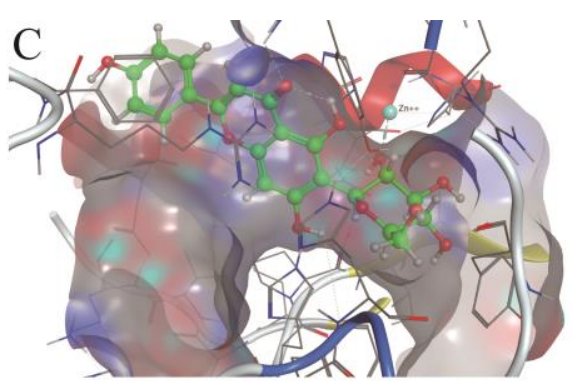

(i: 귬

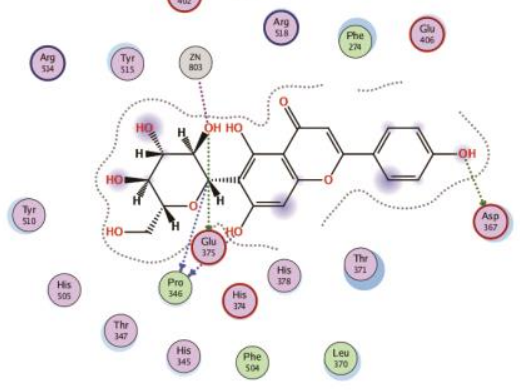

Figure 5. 3D active pocket and 2D interaction of identified optimum conformation against ACE2 (1r4l) (A) Hydroxychloroquine (B) TMDB-00174 (C) Isovitexin (D) Q3G (E) TF. 


\begin{tabular}{|l|l|l|l|}
\hline Protein targets & PDB ID & Drugs & DS (kcal/mol) \\
\hline Angiotensin-converting enzyme 2 & $1 \mathrm{r} 41$ & Hydroxychloroquine & -7.8583 \\
\hline RNA-dependent RNA polymerase from SARS-CoV-2 & $7 \mathrm{bv2}$ & Remdesivir & -9.1084 \\
\hline Main protease from SARS-CoV-2 & $61 \mathrm{u} 7$ & Lopinavir & -8.7147 \\
\hline
\end{tabular}

Table 1. Known effective drugs and protein targets involved in SARS-CoV-2 infection. 


\begin{tabular}{|c|c|c|c|}
\hline Protein targets & TMDB ID & DS (kcal/mol) & Degree \\
\hline \multirow{10}{*}{$61 \mathrm{7}$} & TMDB-00167 & -8.34 & 3 \\
\hline & TMDB-00198 & -7.99 & 3 \\
\hline & TMDB-00228 & -7.95 & 3 \\
\hline & TMDB-00211 & -7.91 & 3 \\
\hline & TMDB-00229 & -7.86 & 3 \\
\hline & TMDB-00033 & -7.83 & 3 \\
\hline & TMDB-00205 & -7.79 & 3 \\
\hline & TMDB-01317 & -7.74 & 3 \\
\hline & TMDB-01285 & -7.74 & 3 \\
\hline & TMDB-00001 & -7.69 & 3 \\
\hline \multirow[t]{10}{*}{$7 b v 2$} & TMDB-00229 & -11.83 & 3 \\
\hline & TMDB-00228 & -11.62 & 3 \\
\hline & TMDB-01430 & -9.77 & 3 \\
\hline & TMDB-00185 & -9.76 & 2 \\
\hline & TMDB-00196 & -9.46 & 3 \\
\hline & TMDB-00285 & -9.33 & 2 \\
\hline & TMDB-01238 & -9.22 & 2 \\
\hline & TMDB-00213 & -9.10 & 3 \\
\hline & TMDB-00202 & -9.06 & 2 \\
\hline & TMDB-01388 & -8.92 & 3 \\
\hline \multirow[t]{10}{*}{$1 \mathrm{r} 41$} & TMDB-00174 & -10.03 & 3 \\
\hline & TMDB-00172 & -9.52 & 1 \\
\hline & TMDB-01309 & -9.26 & 3 \\
\hline & TMDB-00185 & -9.19 & 2 \\
\hline & TMDB-01238 & -9.09 & 2 \\
\hline & TMDB-01317 & -9.08 & 3 \\
\hline & TMDB-01268 & -8.95 & 2 \\
\hline & TMDB-00030 & -8.90 & 3 \\
\hline & TMDB-00196 & -8.82 & 3 \\
\hline & TMDB-01387 & -8.80 & 3 \\
\hline
\end{tabular}

Table 2. Top 10 tea flavonoids for each target 


\begin{tabular}{|c|c|c|c|c|c|c|}
\hline TMDB ID & Structure & Name & Degree & $\mathrm{DS}(\mathrm{kcal} / \mathrm{mol})$ & $\mathrm{DL}$ & $\overline{\mathrm{BS}}$ \\
\hline TMDB- 01238 & & $\begin{array}{l}4,5,7- \\
\text { Trihydroxyflavanone 7- } \\
\text { O-Fructoside (S)-form }\end{array}$ & 2 & $\begin{array}{l}\text { 7bv2: }-9.22 \\
\text { 1r41: }-9.09\end{array}$ & 0.98 & 0.55 \\
\hline TMDB-00185 & & Isovitexin & 2 & $\begin{array}{l}\text { 7bv2: }-9.76 \\
\text { 1r41: }-9.19\end{array}$ & 0.59 & 0.55 \\
\hline TMDB-00196 & & Quercetin 3-glycosides & 3 & $\begin{array}{l}\text { 7bv2: }-9.46 \\
\text { 1r41: }-8.82 \\
\text { 6lu7: }-7.30\end{array}$ & 0.70 & 0.17 \\
\hline TMDB-01317 & & $\begin{array}{l}\text { Epigallocatechin 3-O- } \\
\text { caffeate }\end{array}$ & 3 & $\begin{array}{l}\text { 6lu7: }-7.74 \\
\text { 1r4l: }-9.08 \\
\text { 7bv2: }-7.09\end{array}$ & 0.38 & 0.55 \\
\hline TMDB-00228 & & Cyanidin 3-glycosides & 3 & $\begin{array}{l}\text { 7bv2: }-11.61 \\
\text { 6lu7: }-7.95 \\
\text { 1r4l: }-8.00\end{array}$ & 0.07 & 0.17 \\
\hline TMDB-00229 & & $\begin{array}{l}\text { Delphinidin 3- } \\
\text { glycosides }\end{array}$ & 3 & $\begin{array}{l}\text { 7bv2: }-11.83 \\
\text { 6lu7: }-7.86 \\
\text { 1r4l: }-8.12\end{array}$ & 0.26 & 0.17 \\
\hline
\end{tabular}

Table 3. Overlapping components in top 10 tea flavonoids of each target 
Figures

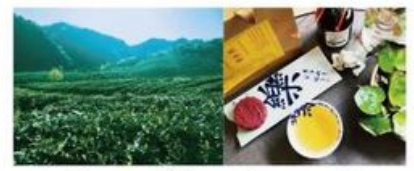

Camellia sinensis

Collection and screening<smiles>c1ccc(C2CCc3ccccc3C2)cc1</smiles>

121 compounds

from tea flavonoids
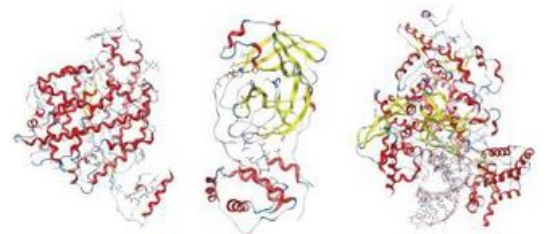

COVID-19-related protein target

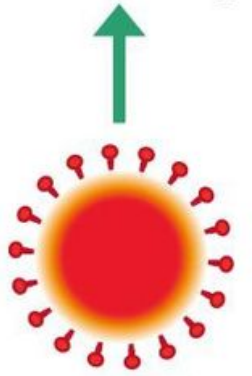

SARS-CoV-2

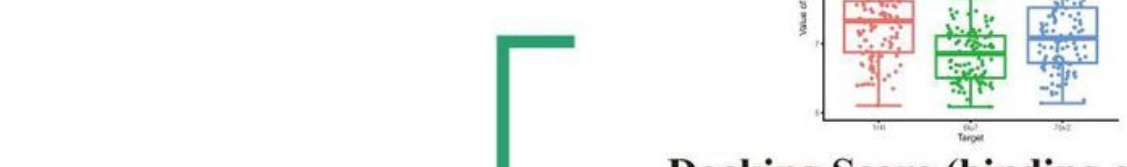

Docking Score (binding energy)

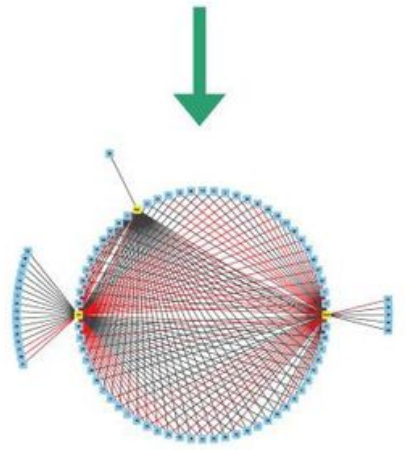

Compounds-targets network
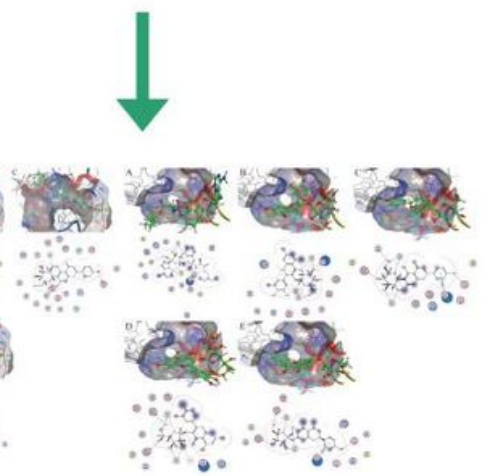

The best conformation and main target

Figure 1

Flow scheme of exploring the inhibitors against multiple SARS-CoV-2 targets 


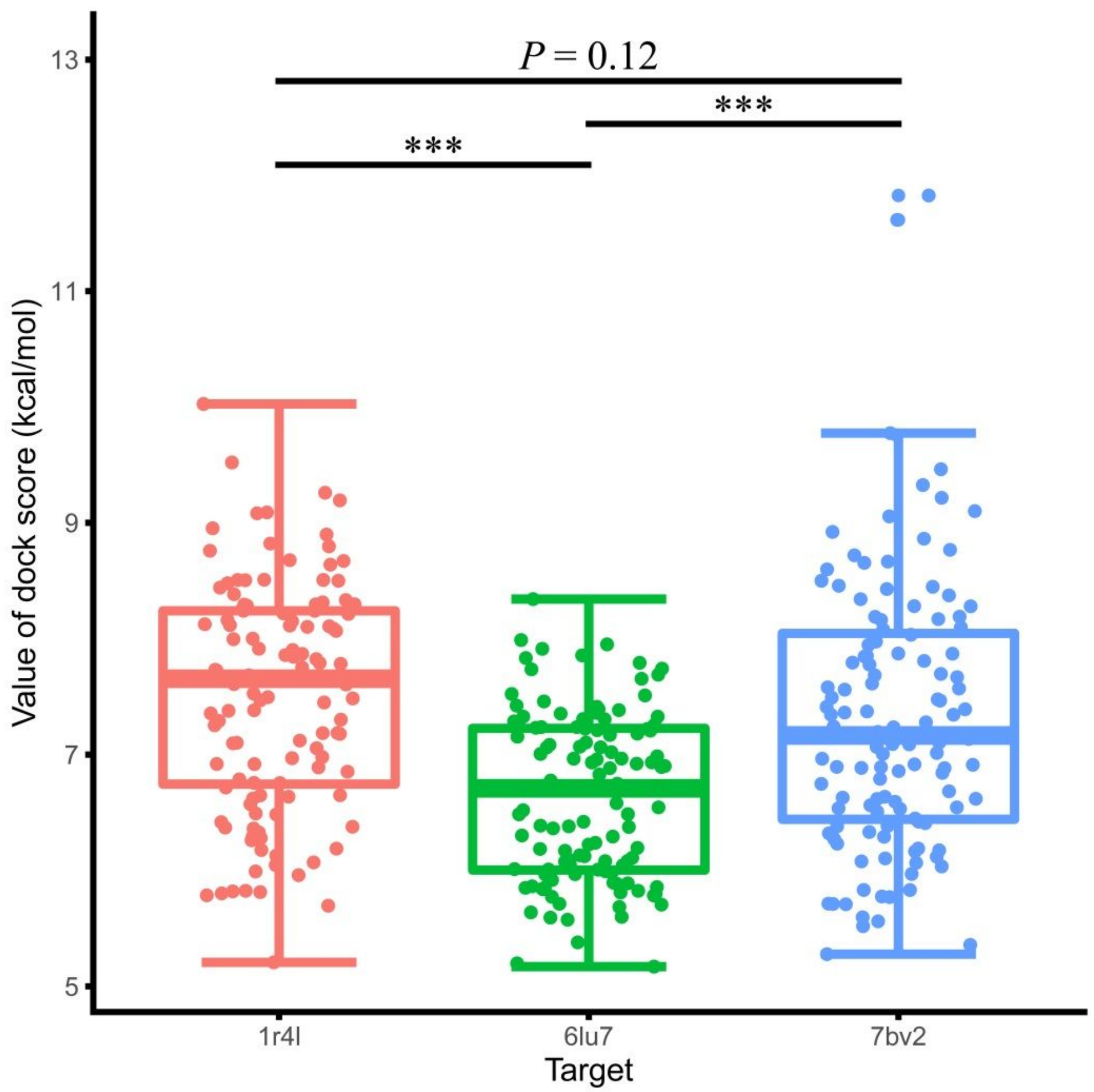

Figure 2

DS absolute value of the compounds-targets interaction. The minimum and maximum values are directly observed from the boxplot; the boxplot center is the median; the boxplot edges represent the 25th and 75th percentiles. Pairwise. t. test ( $p$. adjust. method $=$ "fdr"). *: $p<0.05 ; * \star: p<0.01 ; * \star \star: p<0.001$. 


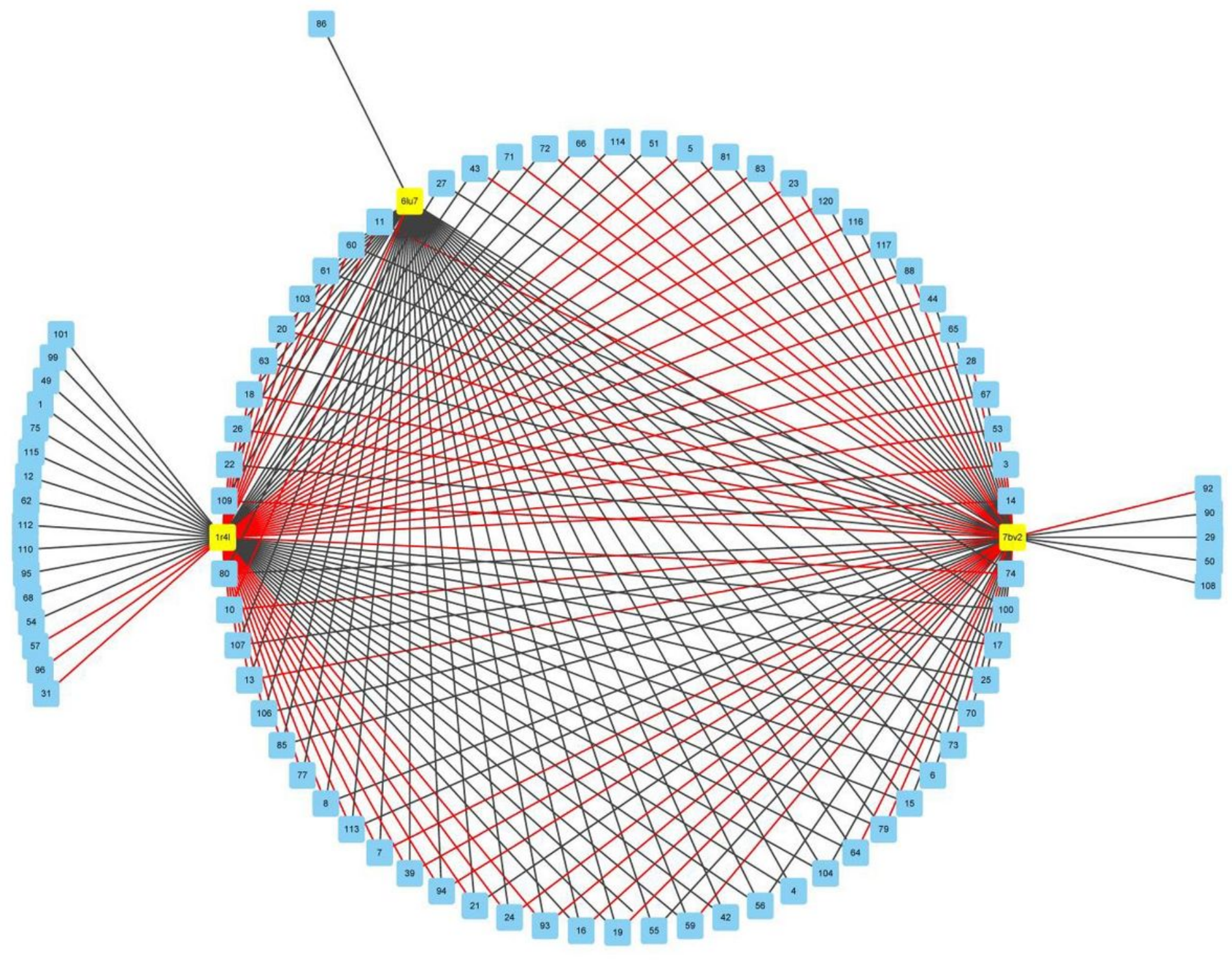

Figure 3

The network model of the compounds-targets interaction. The blue square nodes represent the tea flavonoids, the yellow square nodes represent the protein targets. The red edge denote a strong binding capacity ( $|D S| \geq 8)$, while the black edge denotes a good binding capacity ( $8 \geq|D S| \geq 7)$. 

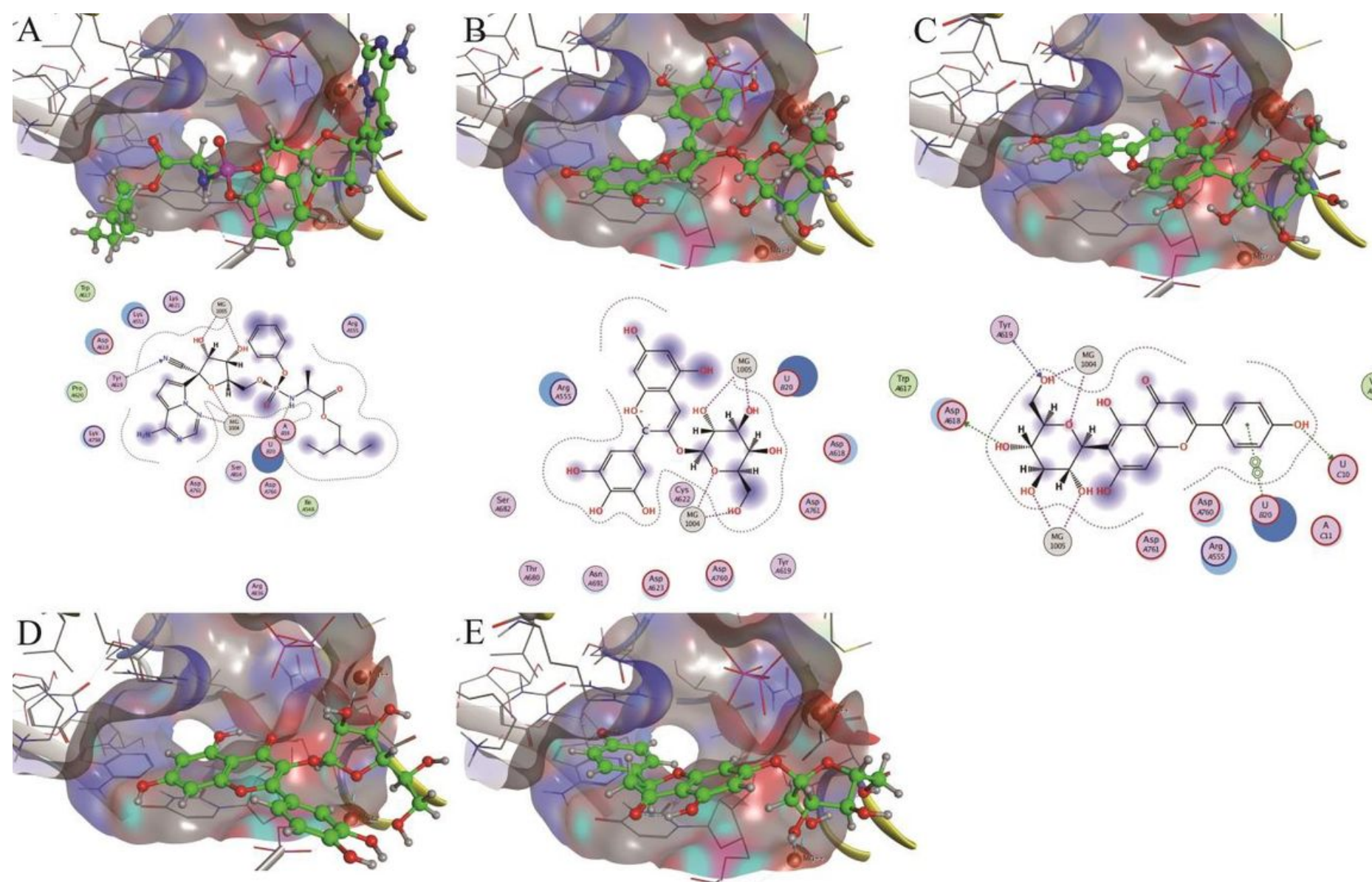
(iii) (iii)
(류)
(200) (중

(iip)
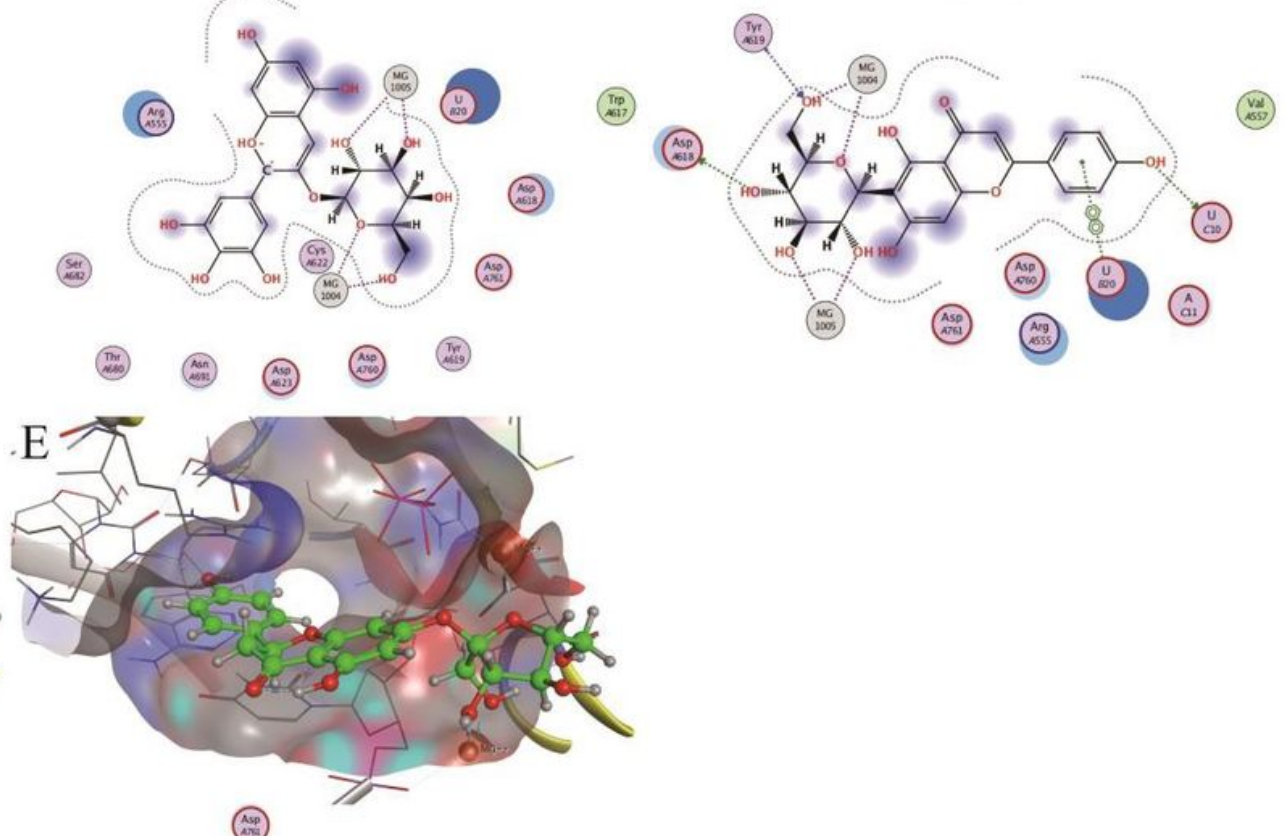

(iㅜㅇ (중

(25)

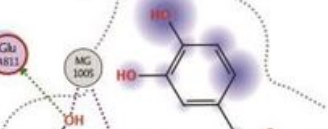

(iin)

(Iiv)
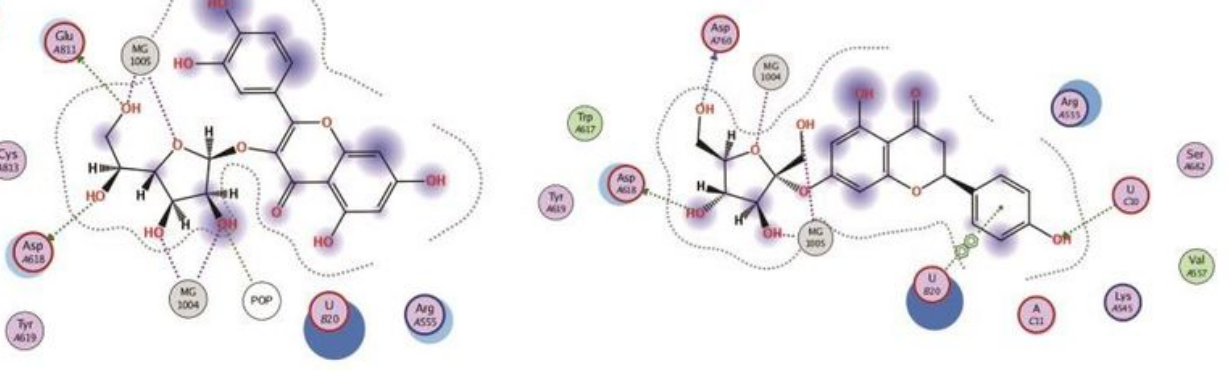

Figure 4

3D active pocket and 2D interaction of identified optimum conformation against RdRp (7bv2). (A) Remdesivir (B) TMDB-00229 (C) Isovitexin (D) Q3G (E) TF. 


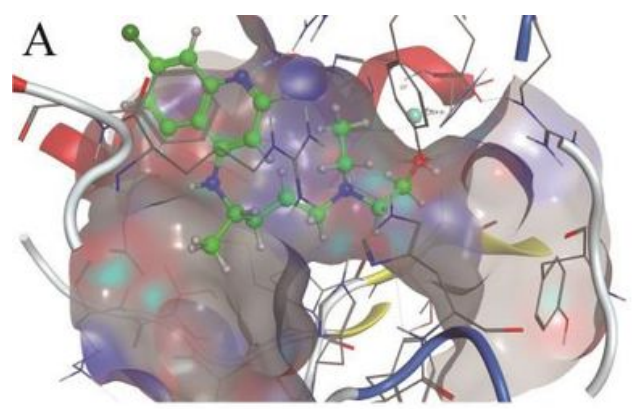

(iii)
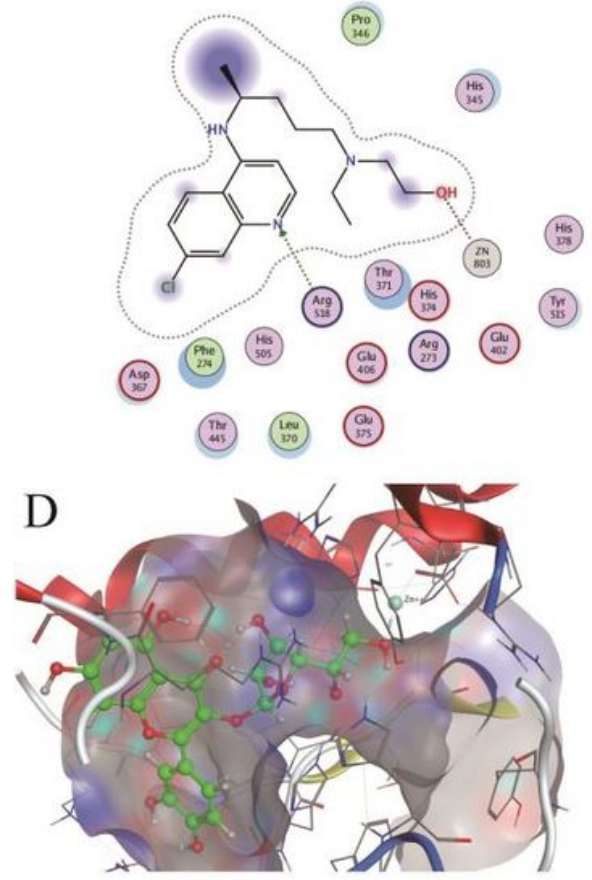

:;)

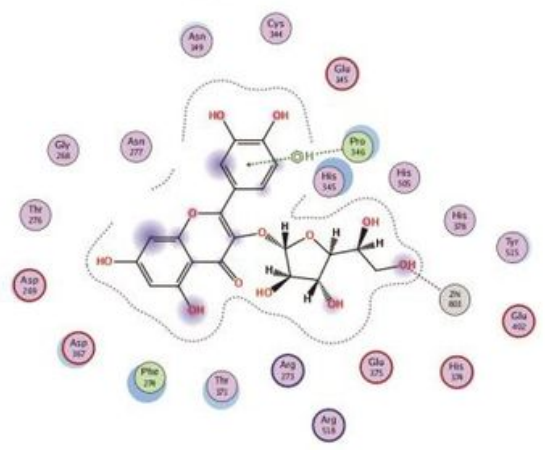

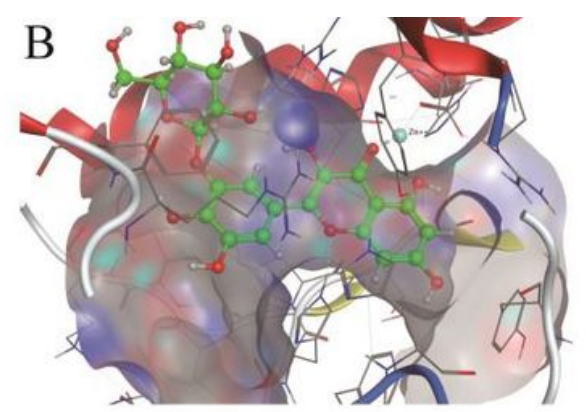

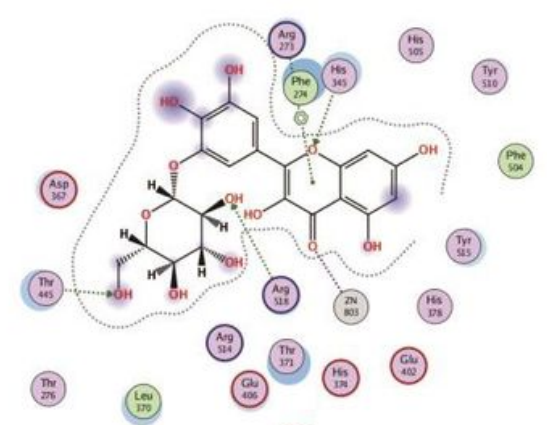

;:

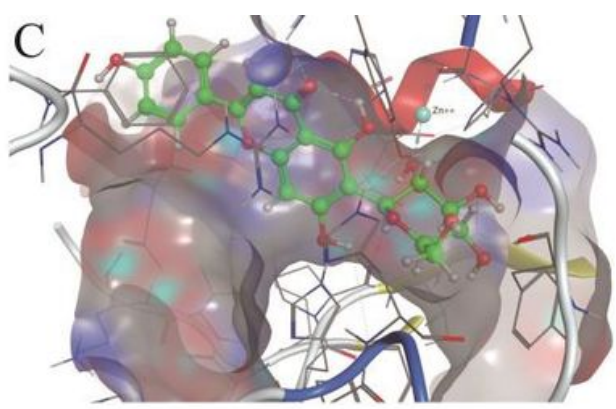

(ㅇ) ; (2)

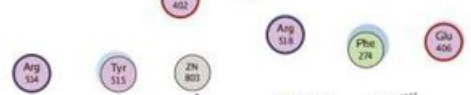

(i:3)

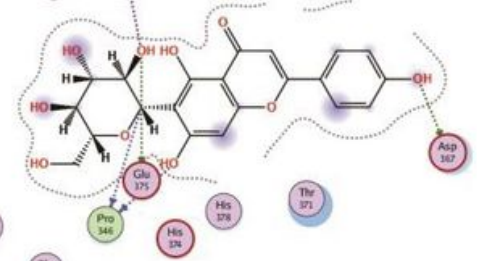

(i:)

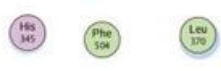

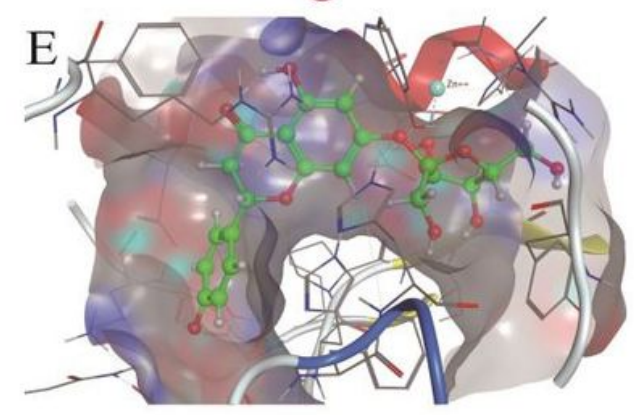

(;)

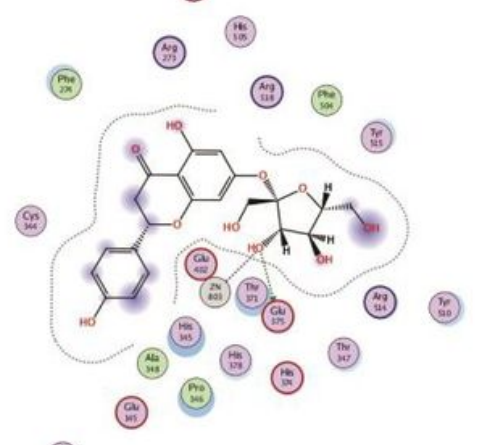

(20)

Figure 5

$3 \mathrm{D}$ active pocket and $2 \mathrm{D}$ interaction of identified optimum conformation against ACE2 (1 r4I) (A) Hydroxychloroquine (B) TMDB-00174 (C) Isovitexin (D) Q3G (E) TF.

\section{Supplementary Files}

This is a list of supplementary files associated with this preprint. Click to download. 